\title{
Correlations Between Mitochondrial Biomarkers without Considering Heteroplasmy Rates Remain Vague
}

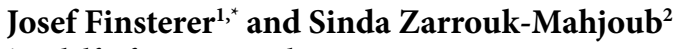 \\ ${ }^{1}$ Rudolfstiftung, Messerli Institute, Veterinary University of Vienna, Austria \\ ${ }^{2}$ University of Tunis El Manar and Genomics Platform, Pasteur Institute of Tunis, Tunisia
}

In a recent article, Lee et al. reported about a study on the relation between magnetic resonance spectroscopy (MRS) and serum biomarkers during acute and chronic stages of a stroke-like episode (SLE) in 13 patients carrying the mtDNA variant m.3243A $>\mathrm{G}$. The study is interesting but has a number of shortcomings.

The main disadvantage of the study is that heteroplasmy rates of the m.3243A $>\mathrm{G}$ variant were not considered as determinants of the correlation between MRS parameters (NNA/Cr, Chol/Cre, NAA/ Chol, lactate) and serum lactate/pyruvate. Since there may be a strong genotype/phenotype correlation, any correlation between serum/ MRS biomarkers can be reliably assessed only when the mutation load is known. Also, the influence of the variability of heteroplasmy rates between tissues on MRS/serum biomarkers correlations should be considered. However, heteroplasmy rates of blood lymphocytes may not correlate with those of brain tissue and heteroplasmy rates may not be the only determinant of the phenotype.

Secondly, it is unclear at which point of a SLE serum/MRS biomarkers were determined. Since stroke-like lesions (SLLs), the morphological equivalent of a SLE, undergo rapid changes over time, the exact interval between onset of a SLE and the investigation should be known. Since MRS findings strongly depend on the stage and volume of a SLL, it should be mentioned if the investigations were repeated at a second or third time point, and if differences between different time points were found. Since no follow-up investigations were carried out and no information about the development of the serum/MRS biomarker relationship over time is available, we do not agree with the statement that serum/MRS biomarkers relationships are useful for monitoring the progression of MELAS [1].

Severity and outcome of SLLs may strongly depend on whether affected patients received nitric-oxide (NO) precursors or not [2]. Those receiving NO-precursors may have a milder course and a more favourable outcome than those without receiving them. This is why the number of patients receiving NO-precursors should be provided?

Lactate concentrations may strongly depend on whether there was previous seizure activity or not [3]. Since epilepsy is a frequent feature of MELAS and strongly influences the outcome and prognosis [4], we should be informed how many of the patients experienced a seizure prior to the examination. Since seizure frequency strongly depends on the quality of seizure control, we should also know which antiepileptic drugs the patients were regularly taking.

Since serum lactate derives from the skeletal muscle and cerebral lactate from the brain, it should be mentioned how many of the included patients had mitochondrial myopathy. It should be also mentioned how many had elevated serum lactate but normal CSF lactate and vice versa.

In summary, we do not agree with the conclusions of the study as long as the influence of heteroplasmy rates, previous seizures, antiepileptic treatment, and disease stage on biomarker correlations

\section{Publication History:}

Received: October 10, 2018

Accepted: October 20, 2018

Published: October 22, 2018

\section{Keywords:}

Cardiac risk score, Quality of life, Hemodialysis

was properly assessed. Presumed homogeneity of the cohort has not been achieved with the settings chosen for this investigation.

\section{Competing Interest}

The authors declare no competing interest.

\section{References}

1. Lee HN, Yoon CS, Lee YM (2018) Correlation of Serum Biomarkers and Magnetic Resonance Spectroscopy in Monitoring Disease Progression in Patients With Mitochondrial Encephalomyopathy, Lactic Acidosis, and Stroke-Like Episodes Due to mtDNA A3243G Mutation. Front Neurol 9: 621.

2. El-Hattab AW, Emrick LT, Hsu JW, Chanprasert S, Almannai M, et al. (2016) Impaired nitric oxide production in children with MELAS syndrome and the effect of arginine and citrulline supplementation. Mol Genet Metab 117: 407-412.

3. Chatzikonstantinou A, Ebert AD, Hennerici MG (2015) Cerebrospinal fluid findings after epileptic seizures. Epileptic Disord 17: 453-459.

4. Lee HN, Eom S, Kim SH, Kang HC, Lee JS, et al. (2016) Epilepsy Characteristics and Clinical Outcome in Patients With Mitochondrial Encephalomyopathy, Lactic Acidosis, and Stroke-Like Episodes (MELAS). Pediatr Neurol 64: 59-65.

*Corresponding Author: Dr. Josef Finsterer, Krankenanstalt Rudolfstiftung, Postfach 20, 1180 Vienna, Austria, Europe, Tel. +43-1-71165-72085, Fax. +43-14781711; Email: fifigs1@yahoo.de

Citation: Finsterer J, Zarrouk-Mahjoub S (2018) Correlations Between Mitochondrial Biomarkers without Considering Heteroplasmy Rates Remain Vague. Int J Community Fam Med 3: 145. https://doi.org/10.15344/2456$3498 / 2018 / 145$

Copyright: (c) 2018 Finsterer et al. This is an open-access article distributed under the terms of the Creative Commons Attribution License, which permits unrestricted use, distribution, and reproduction in any medium, provided the original author and source are credited. 